\title{
On Nonlinear Resonances in Satellite Oscillations
}

\author{
E.A. Kosjakov, T.A. Partalin, A.A. Tikhonov \\ Paper dedicated to memory of Vera Nikolić-Stanojević, \\ Pofessor Emeritus at the State University of Novi Pazar
}

\begin{abstract}
Oscillatory attitude motion of gravity-gradient stabilized spacecraft is under consideration. The spacecraft librational motion is going under the action of the gravitational torque and the other torques of different nature providing disturbing influence in spacecraft oscillations. The perturbation technique is used for approximate analytical research of spacecraft oscillations. A special notation of differential equations of perturbed librational motion for gravity-gradient stabilized spacecraft is derived which is generalization of familiar equations in canonical variations, to the case when potential disturbing forces are operative along with non-potential disturbing forces, usable in a quadratic or cubic approximations. The derived equations proved to be convenient for the analysis of nonlinear spacecraft oscillations with the use of asymptotic methods of nonlinear mechanics and allowed to obtain new effects in nonlinear spacecraft oscillations.
\end{abstract}

Keywords: spacecraft, gravity-gradient torque, librational motion, nonlinear differential equations, perturbation technique, nonlinear resonances

\section{Introduction and preliminaries}

The satellite attitude motion in the neighborhood of stable equilibrium due to the influence of gravity-gradient torque is analyzed. Here and further we call such equilibrium the equilibrium position of gravitational orientation, and the satellite performing librational motion in the vicinity of this position as the gravity-oriented satellite. Satellite librational motion takes place, generally speaking, not only under the action of the gravitational torque but also under the action of the other torques, which are of different nature, providing disturbances in the oscillations of gravityoriented satellite. The investigation of perturbed oscillations is nonlinear problem and it is natural to use a perturbation technique for the approximate analytical solution of this problem. However, perturbation techniques usually involves transition

Manuscript received May 13, 2014. ; accepted October 18, 2014.

E.A. Kosjakov and A.A. Tikhonov are with the Saint Petersburg State University, Russia; T.A. Partalin is with the Sofia university "St. Kliment Ohridski", Bulgaria. 
to new variables, and it is often not easy to construct a transformed system of differential equations of motion. In some cases, these difficulties can be largely overcome by using the method of variation for canonical arbitrary constants. This method is convenient for the construction of differential equations of perturbed motion for mechanical systems whose state can be described by canonical equations. For example, in [1], [2], the method of variation of canonical arbitrary constants combined with an averaging technique was successfully used for investigation of gravity-oriented satellite nonlinear oscillations under the action of the gravity-gradient torque, taken in quadratic approximation.

A special notation is suggested in [3] to write the differential equations of perturbed librational motion of gravity-oriented satellite. These equations generalize the familiar equations used in [1] and [2] to the case when potential disturbing forces are operative along with nonpotential disturbing forces, usable in a quadratic approximation. The differential equations derived in [3] proved to be convenient for their further analysis by means of asymptotic methods of nonlinear mechanics and allowed to obtain new results.

Thus, in [4] and [5] nonlinear vibrations of a gravity-oriented charged satellite in a superposition of terrestrial gravitational and magnetic (in the dipole approximation) fields were studied. In this case 22 parametric and 4 internal nonlinear resonances due to quadratic nonlinear terms were discovered in the region of gravitational orientation. With the use of the averaging technique nonlinear vibrations of a gravity-oriented charged satellite were investigated in the nonresonance case and in conditions of revealed resonances by constructing the sets of first integrals with further integration of differential equations and solution interpretation on the amplitude-phase planes. On the basis of this research a number of new results were obtained. In particular, the phenomena of "pumping" the energy of the longitudinal oscillations of a charged satellite into transversal and vice versa were revealed. The possible stationary modes providing vibrations of a charged satellite with the least deviation from the gravitational orientation were revealed.

On the basis of the same differential equations derived in [3], but in a more precise quadrupole approximation of the Earth's magnetic field (EMF), the nonlinear vibrations of a gravity-oriented charged satellite in the superposition of the gravitational and magnetic fields were considered in [6]. A number of qualitatively new effects were found. In particular, it was found that the number of realizable resonances depends on the altitude of the satellite orbit. Such dependence, as follows from the analysis of [4], does not appear in the dipole approximation of the EMF and at first was detected while using the quadrupole approximation of the EMF and taking into account the significant dependence of the EMF magnetic induction on the daily rotation of the Earth.

Later, on the basis of equations derived in [3], there was developed a general method for investigating the librational motion of a satellite under the influence of the perturbing torque of a general form in the class of nonlinear quadratic functions 
with respect to small angles of orientation of a satellite and their time derivatives. In [7] the spectral structure of this perturbing torque was cleared up, thus allowing to detect possible parametric and internal resonances. In [8] 63 resonance combinations of the unperturbed natural satellite frequencies there were found realizable in the domain of gravitational orientation, and the corresponding resonance curves were constructed in the satellite inertial parameters plane. 520 multiple resonances within the domain of gravitational orientation of the satellite were also identified and the corresponding values of inertia parameters were found. In the papers [9] and [10] the system of differential equations of the satellite perturbed motion was averaged over explicitly appearing dimensionless time in the nonresonance case and in the identified resonances. The structure of obtained averaged differential systems was made clear, the coefficients of the averaged equations as functions of the initial parameters of the problem were obtained and the unified notation of averaged differential equations of satellite perturbed motion were obtained for all resonance cases. The satellite oscillations were investigated in the nonresonance case and in all possible resonances. The conditions of damped, limited or divergent satellite oscillations were obtained. These results allowed researchers of specific applied problems in dynamics of gravity-oriented satellites to understand what resonances can occur in different cases of disturbances in the class of quadratic functions, and what effects can these resonances cause.

At the same time many examples of well-known problems of nonlinear mechanics demonstrate the importance of taking into account nonlinear terms of the third order of smallness. In full measure it is valid with respect to nonlinear vibration problems in conditions of internal resonances.

\section{Main result}

In this paper we solve the problem of obtaining such differential equations of perturbed librational motion of gravity-oriented satellite, which allows to take into account not only the influence of quadratic but also cubic nonlinear terms. Retaining the notation of papers mentioned above, we write differential equations of satellite perturbed oscillations in the following form: 


$$
\begin{aligned}
x_{i}{ }^{\prime} & =-\frac{\partial\left(H_{1}+H_{2}\right)}{\partial v_{i}}+2(-1)^{i} \sqrt{x_{i}}\left(\frac{\sigma_{1,3-i}}{\sigma_{1}} \widetilde{M}_{B x}^{(3)} \sin \tau_{i}-\frac{\sigma_{2,3-i}}{\sigma_{2}} \widetilde{M}_{B z}^{(3)} \cos \tau_{i}\right)+ \\
& +2 \sqrt{2}(-1)^{i} \frac{A \sigma_{2,3-i}}{C \sqrt{B} \sigma_{2} k_{3}} \sqrt{x_{i} x_{3}} \widetilde{M}_{B x}^{(2)} \cos \tau_{i} \sin \tau_{3}- \\
& -2(-1)^{i} \frac{B \sigma_{2,3-i}}{C \sigma_{2}} \sqrt{x_{i}}\left(\sigma_{21} \sqrt{x_{1}} \cos \tau_{1}+\sigma_{22} \sqrt{x_{2}} \cos \tau_{2}\right) \widetilde{M}_{B y}^{(2)} \cos \tau_{i}, \\
v_{i}^{\prime} & =\frac{\partial\left(H_{1}+H_{2}\right)}{\partial x_{i}}+\frac{(-1)^{i}}{k_{i} \sqrt{x_{i}}}\left(\frac{\sigma_{1,3-i}}{\sigma_{1}} \widetilde{M}_{B x}^{(3)} \cos \tau_{i}+\frac{\sigma_{2,3-i}}{\sigma_{2}} \widetilde{M}_{B z}^{(3)} \sin \tau_{i}\right)+ \\
& +\sqrt{2}(-1)^{i} \frac{A \sigma_{2,3-i} \sqrt{x_{3}}}{C \sqrt{B} \sigma_{2} k_{i} k_{3} \sqrt{x_{i}}} \widetilde{M}_{B x}^{(2)} \sin \tau_{i} \sin \tau_{3}+ \\
& +(-1)^{i} \frac{B \sigma_{2,3-i}}{C \sigma_{2} k_{i} \sqrt{x_{i}}}\left(\sigma_{21} \sqrt{x_{1}} \cos \tau_{1}+\sigma_{22} \sqrt{x_{2}} \cos \tau_{2}\right) \widetilde{M}_{B y}^{(2)} \sin \tau_{i}, \\
x_{3}{ }^{\prime} & =-\frac{\partial\left(H_{1}+H_{2}\right)}{\partial v_{3}}+\sqrt{2 B x_{3}} \widetilde{M}_{B y}^{(3)} \cos \tau_{3}- \\
& -\frac{C \sqrt{2 x_{3}}}{\sqrt{B}}\left(\sigma_{21} \sqrt{x_{1}} \cos \tau_{1}+\sigma_{22} \sqrt{x_{2}} \cos \tau_{2}\right) \widetilde{M}_{B z}^{(2)} \cos \tau_{3}, \\
v_{3}^{\prime}= & \frac{\partial\left(H_{1}+H_{2}\right)}{\partial x_{3}}-\frac{\sqrt{B}}{k_{3} \sqrt{2 x_{3}}} \widetilde{M}_{B y}^{(3)} \sin \tau_{3}+ \\
+ & \frac{C}{k_{3} \sqrt{2 B x_{3}}}\left(\sigma_{21} \sqrt{x_{1}} \cos \tau_{1}+\sigma_{22} \sqrt{x_{2}} \cos \tau_{2}\right) \widetilde{M}_{B z}^{(2)} \sin \tau_{3} .
\end{aligned}
$$

Here $i=1,2 ; A, B, C$ are the satellite principal central moments of inertia; Hamiltonian $H_{1}$ contains the terms of the 3 -rd order of smallness, $H_{2}$ contains the terms of the 4-th order of smallness, $\widetilde{M}_{B x}^{(2)}, \widetilde{M}_{B y}^{(2)}, \widetilde{M}_{B z}^{(2)}$ are the expressions for dimensionless disturbing torque projections $\widetilde{M}_{B x}, \widetilde{M}_{B y}, \widetilde{M}_{B z}$ respectively, containing terms up to the second order of smallness with respect to $\sqrt{x_{1}}, \sqrt{x_{2}}, \sqrt{x_{3}}$, and $\widetilde{M}_{B x}^{(3)}, \widetilde{M}_{B y}^{(3)}, \widetilde{M}_{B z}^{(3)}$ are the analogical expressions, containing terms up to the third order of smallness.

The problem of investigation of gravity-oriented satellite oscillations is a nonlinear mechanical problem where the analysis of possible resonance effects is of great interest [1], [2]. At the same time the equations (1) are convenient both for finding out resonance cases and for further analytical investigation of satellite resonance oscillations with the help of asymptotic methods. As is generally known, with the exception of very degenerate cases, only resonances of the 2-nd, 3-rd and 4-th orders are the most important [11]. With due account of nonlinear terms of the 3-rd order of smallness in the problems of satellite attitude dynamics one can reveal internal resonances of the 4 -th order and finish with searching for the most important resonances in the above mentioned sense. For the case when the disturbing torque $\vec{M}_{B}$ is absent, the following resonant combinations of the 4 -th order for natural frequencies 
of gravity-oriented satellite are obtained:

$$
2 k_{1}-2 k_{3}=0,3 k_{1}-k_{2}=0,2 k_{2}-2 k_{3}=0, k_{1}+k_{2}-2 k_{3}=0, k_{1}-k_{2}+2 k_{3}=0 \text {. }
$$

All of them may realize in the domain where the sufficient conditions $B>A>C$ [2] for the stability of gravity-gradient satellite equilibrium are valid. At the same time the fulfilment of sufficient conditions for stability does not guarantee the satellite oscillation amplitudes staying within the acceptable from a practical point of view range. Therefore particularly relevant in problems of this kind are the analysis of resonance effects [2] and the issue of satellite oscillation amplitudes in possible resonances.

With the use of averaging technique the differential equations of satellite perturbed oscillations are analyzed in nonresonance case and for all resonances of gravitational nature, including resonances (2). The complete sets of first integrals are constructed for averaged differential equations in each resonance case. With the use of these integrals the solutions of differential systems are interpreted in amplitudephase planes. It was revealed that the amplitudes of resonance oscillations are limited. This conclusion was verified numerically. The upper bounds of oscillation amplitudes for all resonance cases are obtained by computer modeling on the basis of initial nonlinear differential system.

\section{Acknowledgement}

The reported study was supported by the Saint Petersburg State University, Project No. 6.0.8.2010 and by the Russian Foundation for Basic Research, Grant Nos. 1301-00347-a and 13-01-00376-a.

\section{References}

[1] J. V. Breakwell and R. PRINGle JR., Non-linear resonances affecting gravitygradient stability, Astrodynamics, Gauthier-Villars, Paris, 1966, 305-325.

[2] V. V. Beletsky, Motion of a Satellite about its Center of Mass in the Gravitational Field, MSU Publishers, Moscow, 1975.

[3] A. A. Tiknonov, A Form of Differential Equations for Disturbed Motion of GravityOriented Rigid Body, Leningrad University Mechanics Bulletin. 1 (1990), 15-18.

[4] A. A. Tikhonov, Nonlinear Resononce Vibrations of a Charged Rigid Body in a Nonstationary Magnetic Field. Part 1, Leningrad University Mechanics Bulletin. 3 (1992), 12-19.

[5] A. A. Tikhonov, Nonlinear Resononce Vibrations of a Charged Rigid Body in a Nonstationary Magnetic Field. Part 2, Leningrad University Mechanics Bulletin. 4 (1992), 28-34. 
[6] A. A. Tikhonov and O. Yu. Alekhina, Nonlinear Resonances in the Problem of the Satellite's Librational Motion in Quadrupole Approximation of Geomagnetic Field, Problems of analytical mechanics and stability theory - the book of selected articles in memory of academician V.V.Rumyantsev, Fizmatlit, Moscow 2009, 148-160.

[7] A. A. Tikhonov, Resononce Phenomena in Oscillations of a Gravity-Oriented Rigid Body. Part I. The Spectral Structure of a Perturbation Moment, St.Petersburg University Mechanics Bulletin. 2, 15-23 (1997)

[8] A. A. Tikhonov, Resononce Phenomena in Oscillations of a Gravity-Oriented Rigid Body. Part II. The Realizable Resononces, St.Petersburg University Mechanics Bulletin. 3, 14-22 (1997)

[9] A. A. Tiknonov, Resononce Phenomena in Oscillations of a Gravity-Oriented Rigid Body. Part III. The Averaged Equations. Single-Frequency Resononces, St.Petersburg University Mechanics Bulletin. 3, 42-51 (1998)

[10] A. A. Tikhonov, Resononce Phenomena in Oscillations of a Gravity-Oriented Rigid Body. Part IV. Multy-Frequency Resononces, St.Petersburg University Mechanics Bulletin. 1, 131-137 (2000)

[11] A. L. Kunitsyn, The resonance stabilization of a class of unstable systems, Journal of Applied Mathematics and Mechanics. 75 (2011), 508-510. 